PROCEEDINGS OF THE

AMERICAN MATHEMATICAL SOCIETY

Volume 130, Number 2, Pages 565-576

S 0002-9939(01)06079-8

Article electronically published on June 22, 2001

\title{
EXTENSIONS AND EXTREMALITY OF RECURSIVELY GENERATED WEIGHTED SHIFTS
}

\author{
RAÚl E. CURTO, IL BONG JUNG, AND WOO YOUNG LEE
}

(Communicated by Joseph A. Ball)

\begin{abstract}
Given an $n$-step extension $\alpha: x_{n}, \cdots, x_{1},\left(\alpha_{0}, \cdots, \alpha_{k}\right)^{\wedge}$ of a recursively generated weight sequence $\left(0<\alpha_{0}<\cdots<\alpha_{k}\right)$, and if $W_{\alpha}$ denotes the associated unilateral weighted shift, we prove that

$$
W_{\alpha} \text { is subnormal } \Longleftrightarrow \begin{cases}W_{\alpha} \text { is }\left(\left[\frac{k+1}{2}\right]+1\right) \text {-hyponormal } & (n=1), \\ W_{\alpha} \text { is }\left(\left[\frac{k+1}{2}\right]+2\right) \text {-hyponormal } & (n>1) .\end{cases}
$$

In particular, the subnormality of an extension of a recursively generated weighted shift is independent of its length if the length is bigger than 1. As a consequence we see that if $\alpha(x)$ is a canonical rank-one perturbation of the recursive weight sequence $\alpha$, then subnormality and $k$-hyponormality for $W_{\alpha(x)}$ eventually coincide. We then examine a converse - an "extremality" problem: Let $\alpha(x)$ be a canonical rank-one perturbation of a weight sequence $\alpha$ and assume that $(k+1)$-hyponormality and $k$-hyponormality for $W_{\alpha(x)}$ coincide. We show that $\alpha(x)$ is recursively generated, i.e., $W_{\alpha(x)}$ is recursive subnormal.
\end{abstract}

\section{INTRODUCTION}

Let $\mathcal{H}$ be a separable infinite dimensional complex Hilbert space and let $\mathcal{L}(\mathcal{H})$ be the algebra of bounded linear operators on $\mathcal{H}$. An operator $T \in \mathcal{L}(\mathcal{H})$ is said to be normal if $T^{*} T=T T^{*}$ and hyponormal if $T^{*} T \geq T T^{*}$. Given a bounded sequence of positive numbers $\alpha: \alpha_{0}, \alpha_{1}, \cdots$ (called weights), the (unilateral) weighted shift $W_{\alpha}$ associated with $\alpha$ is the operator on $\ell^{2}\left(\mathbb{Z}_{+}\right)$defined by $W_{\alpha} e_{n}:=\alpha_{n} e_{n+1}$ for all $n \geq 0$, where $\left\{e_{n}\right\}_{n=0}^{\infty}$ is the canonical orthonormal basis for $\ell^{2}\left(\mathbb{Z}_{+}\right)$. It is straightforward to check that $W_{\alpha}$ can never be normal, and that $W_{\alpha}$ is hyponormal if and only if $\alpha_{n} \leq \alpha_{n+1}$ for all $n \geq 0$. The Bram-Halmos criterion for subnormality states that an operator $T$ is subnormal if and only if

$$
\sum_{i, j}\left(T^{i} x_{j}, T^{j} x_{i}\right) \geq 0
$$

Received by the editors July 14, 2000.

1991 Mathematics Subject Classification. Primary 47B20, 47B37; Secondary 47-04, 47A57, $15 \mathrm{~A} 57$.

Key words and phrases. Extensions of weighted shifts, recursively generated shifts, $k$ hyponormality.

The work of the first-named author was partially supported by NSF research grants DMS9401455 and DMS-9800931.

The work of the second-named author was partially supported by KOSEF, research grant 2000-1-10100-002-3.

The work of the third-named author was partially supported by the Brain Korea 21 Project. 
for all finite collections $x_{0}, x_{1}, \cdots, x_{k} \in \mathcal{H}$ ([B] $]$, [Con, III.1.9]). Using Choleski's algorithm for operator matrices, it is easy to see that this is equivalent to the following positivity test:

$$
\left(\begin{array}{cccc}
I & T^{*} & \ldots & T^{* k} \\
T & T^{*} T & \ldots & T^{* k} T \\
\vdots & \vdots & \ddots & \vdots \\
T^{k} & T^{*} T^{k} & \ldots & T^{* k} T^{k}
\end{array}\right) \geq 0 \quad(\text { all } k \geq 1)
$$

Condition (0.1) provides a measure of the gap between hyponormality and subnormality, and $k$-hyponormality has been introduced and studied in an attempt to bridge that gap ([At], Cu1, Cu2, [CF1, [CF2], [CF3], [CL1], [CMX], [McCP]). In fact, the positivity condition (0.1) for $k=1$ is equivalent to the hyponormality of $T$, while subnormality requires the validity of (0.1) for all $k$. If we denote by $[A, B]:=A B-B A$ the commutator of two operators $A$ and $B$, and if we define $T$ to be $k$-hyponormal whenever the $k \times k$ operator matrix

$$
M_{k}(T):=\left(\left[T^{* j}, T^{i}\right]\right)_{i, j=1}^{k}
$$

is positive, or equivalently, the $(k+1) \times(k+1)$ operator matrix in $(0.1)$ is positive, then the Bram-Halmos criterion can be rephrased as saying that $T$ is subnormal if and only if $T$ is $k$-hyponormal for every $k \geq 1$ ([CMX]).

If $W_{\alpha}$ is the weighted shift with weight sequence $\alpha=\left\{\alpha_{n}\right\}_{n=0}^{\infty}$, then the moments of $W_{\alpha}$ are usually defined by $\beta_{0}:=1, \beta_{n+1}:=\alpha_{n} \beta_{n}(n \geq 0)$ [Shi]; however, we reserve this term for the sequence $\gamma_{n}:=\beta_{n}^{2}(n \geq 0)$. A criterion for $k$-hyponormality can be given in terms of moments ([Cu1, Theorem 4]): if we build a $(k+1) \times(k+1)$ Hankel matrix $A(n ; k)$ by

$$
A(n ; k):=\left(\begin{array}{cccc}
\gamma_{n} & \gamma_{n+1} & \ldots & \gamma_{n+k} \\
\gamma_{n+1} & \gamma_{n+2} & \ldots & \gamma_{n+k+1} \\
\vdots & \vdots & & \vdots \\
\gamma_{n+k} & \gamma_{n+k+1} & \ldots & \gamma_{n+2 k}
\end{array}\right) \quad(n \geq 0)
$$

then

$$
W_{\alpha} \text { is } k \text {-hyponormal } \Longleftrightarrow A(n ; k) \geq 0 \quad(n \geq 0) .
$$

In [Sta], J. Stampfli showed that given $\alpha: \sqrt{a}, \sqrt{b}, \sqrt{c}$ with $0<a<b<c$, there always exists a subnormal completion of $\alpha$, but that for $\alpha: \sqrt{a}, \sqrt{b}, \sqrt{c}, \sqrt{d}$ $(a<b<c<d)$ such a subnormal completion may not exist.

There are instances where $k$-hyponormality implies subnormality for weighted shifts. For example, in CF3] it was shown that if $\alpha(x): \sqrt{x},(\sqrt{a}, \sqrt{b}, \sqrt{c})^{\wedge}(a<$ $b<c)$, then $W_{\alpha(x)}$ is 2-hyponormal if and only if it is subnormal: more concretely, $W_{\alpha(x)}$ is 2-hyponormal if and only if

$$
\sqrt{x} \leq H_{2}(\sqrt{a}, \sqrt{b}, \sqrt{c}):=\sqrt{\frac{a b(c-b)}{(b-a)^{2}+b(c-b)}},
$$

in which case $W_{\alpha(x)}$ is subnormal. In this paper we extend the above result to weight sequences of the form $\alpha: x_{n}, \cdots, x_{1},\left(\alpha_{0}, \cdots, \alpha_{k}\right)^{\wedge}$ with $0<\alpha_{0}<\cdots<\alpha_{k}$. Our main results are as follows. 
Extensions of Recursively Generated Weighted Shifts. If $\alpha: x_{n}, \cdots, x_{1}$, $\left(\alpha_{0}, \cdots, \alpha_{k}\right)^{\wedge}$, then

$$
W_{\alpha} \text { is subnormal } \Longleftrightarrow \begin{cases}W_{\alpha} \text { is }\left(\left[\frac{k+1}{2}\right]+1\right) \text {-hyponormal } & (n=1), \\ W_{\alpha} \text { is }\left(\left[\frac{k+1}{2}\right]+2\right) \text {-hyponormal } & (n>1) .\end{cases}
$$

In particular, the above theorem shows that the subnormality of an extension of the recursive shift is independent of its length if the length is bigger than 1.

Canonical Rank-One Perturbations. Let $\alpha \equiv\left\{\alpha_{n}\right\}_{n=0}^{\infty}=\left(\alpha_{0}, \cdots, \alpha_{k}\right)^{\wedge}$. If $W_{\alpha^{\prime}}$ is a perturbation of $W_{\alpha}$ at the $j$-th weight, then

$$
W_{\alpha^{\prime}} \text { is subnormal } \Longleftrightarrow \begin{cases}W_{\alpha^{\prime}} \text { is }\left(\left[\frac{k+1}{2}\right]+1\right) \text {-hyponormal } & (j=0), \\ W_{\alpha^{\prime}} \text { is }\left(\left[\frac{k+1}{2}\right]+2\right) \text {-hyponormal } & (j \geq 1) .\end{cases}
$$

Extremality Criterion. Let $\alpha(x)$ be a canonical rank-one perturbation of a weight sequence $\alpha$. If $(k+1)$-hyponormality and $k$-hyponormality for $W_{\alpha(x)}$ coincide, then $\alpha(x)$ is recursively generated, i.e., $W_{\alpha(x)}$ is recursive subnormal.

\section{Extensions of RECURSIVELY GENERATED SHIFTS}

C. Berger's characterization of subnormality for unilateral weighted shifts (cf. [Hal, Con III.8.16]) states that $W_{\alpha}$ is subnormal if and only if there exists a Borel probability measure $\mu$ support in $\left[0,\left\|W_{\alpha}\right\|^{2}\right]$, with $\left\|W_{\alpha}\right\|^{2} \in \operatorname{supp} \mu$, such that

$$
\gamma_{n}=\int t^{n} d \mu(t) \quad \text { for all } n \geq 0 \text {. }
$$

Given an initial segment of weights $\alpha: \alpha_{0}, \cdots \alpha_{m}$, the sequence $\hat{\alpha} \in \ell^{\infty}\left(\mathbb{Z}_{+}\right)$such that $\hat{\alpha}_{i}=\alpha_{i}(i=0, \cdots, m)$ is said to be recursively generated by $\alpha$ if there exist $r \geq 1$ and $\varphi_{0}, \cdots, \varphi_{r-1} \in \mathbb{R}$ such that

$$
\gamma_{n+r}=\varphi_{0} \gamma_{n}+\cdots+\varphi_{r-1} \gamma_{n+r-1} \text { for all } n \geq 0,
$$

where $\gamma_{0}:=1, \gamma_{n}:=\hat{\alpha}_{0}^{2} \cdots \hat{\alpha}_{n-1}^{2}(n \geq 1)$. In this case the weighted shift $W_{\hat{\alpha}}$ with a weight sequence $\hat{\alpha}$ is said to be recursively generated (or simply recursive). If

$$
g(t):=t^{r}-\left(\varphi_{r-1} t^{r-1}+\cdots+\varphi_{0}\right)
$$

then $g$ has $r$ distinct real roots $0 \leq s_{0}<\cdots<s_{r-1}$ ([CF2, Theorem 3.9]). Let

$$
V:=\left(\begin{array}{cccc}
1 & 1 & \ldots & 1 \\
s_{0} & s_{1} & \ldots & s_{r-1} \\
\vdots & \vdots & & \vdots \\
s_{0}^{r-1} & s_{1}^{r-1} & \ldots & s_{r-1}^{r-1}
\end{array}\right)
$$

and let

$$
\left(\begin{array}{c}
\rho_{0} \\
\vdots \\
\rho_{r-1}
\end{array}\right):=V^{-1}\left(\begin{array}{c}
\gamma_{0} \\
\vdots \\
\gamma_{r-1}
\end{array}\right)
$$

If $W_{\hat{\alpha}}$ is a recursively generated subnormal shift, then the Berger measure of $W_{\hat{\alpha}}$ is of the form

$$
\mu:=\rho_{0} \delta_{s_{0}}+\cdots+\rho_{r-1} \delta_{r-1} .
$$


Given an initial segment of weights

$$
\alpha: \alpha_{0}, \cdots, \alpha_{2 k} \quad(k \geq 0),
$$

suppose $\hat{\alpha} \equiv\left(\alpha_{0}, \cdots, \alpha_{2 k}\right)^{\wedge}$, i.e., $\hat{\alpha}$ is recursively generated by $\alpha$. Write

$$
\mathbf{v}_{n}:=\left(\begin{array}{c}
\gamma_{n} \\
\vdots \\
\gamma_{n+k}
\end{array}\right) \quad(0 \leq n \leq k+1)
$$

Then $\left\{\mathbf{v}_{0}, \cdots, \mathbf{v}_{k+1}\right\}$ is linearly dependent in $\mathbb{R}^{k+1}$. Now the rank of $\alpha$ is defined by the smallest integer $i(1 \leq i \leq k+1)$ such that $\mathbf{v}_{i}$ is a linear combination of $\mathbf{v}_{0}, \cdots, \mathbf{v}_{i-1}$. Since $\left\{\mathbf{v}_{0}, \cdots, \mathbf{v}_{i-1}\right\}$ is linearly independent, there exists a unique $i$ tuple $\varphi \equiv\left(\varphi_{0}, \cdots, \varphi_{i-1}\right) \in \mathbb{R}^{i}$ such that $\mathbf{v}_{i}=\varphi_{0} \mathbf{v}_{0}+\cdots+\varphi_{i-1} \mathbf{v}_{i-1}$, or equivalently,

$$
\gamma_{j}=\varphi_{i-1} \gamma_{j-1}+\cdots+\varphi_{0} \gamma_{j-i} \quad(i \leq j \leq k+i),
$$

which says that $\left(\alpha_{0}, \cdots, \alpha_{k+i}\right)$ is recursively generated by $\left(\alpha_{0}, \cdots, \alpha_{i}\right)$. In this case, $W_{\alpha}$ is said to be $i$-recursive (cf. [CF3, Definition 5.14]).

We begin with:

Lemma 1.1 ([CF2, Propositions 2.3, 2.6, and 2.7]). Let $A, B \in M_{n}(\mathbb{C}), \tilde{A}, \tilde{B} \in$ $M_{n+1}(\mathbb{C})(n \geq 1)$ be such that

$$
\tilde{A}=\left(\begin{array}{ll}
A & * \\
* & *
\end{array}\right) \quad \text { and } \quad \tilde{B}=\left(\begin{array}{cc}
* & * \\
* & B
\end{array}\right) .
$$

Then we have:

(i) If $A \geq 0$ and if $\tilde{A}$ is a flat extension of $A$ (i.e., $\operatorname{rank}(\tilde{A})=\operatorname{rank}(A))$, then $\tilde{A} \geq 0$.

(ii) If $A \geq 0$ and $\tilde{A} \geq 0$, then $\operatorname{det}(A)=0$ implies $\operatorname{det}(\tilde{A})=0$.

(iii) If $B \geq 0$ and $\tilde{B} \geq 0$, then $\operatorname{det}(B)=0$ implies $\operatorname{det}(\tilde{B})=0$.

Lemma 1.2. If $\alpha \equiv\left(\alpha_{0}, \cdots, \alpha_{k}\right)^{\wedge}$, then

$$
W_{\alpha} \text { is subnormal } \Longleftrightarrow W_{\alpha} \text { is }\left(\left[\frac{k}{2}\right]+1\right) \text {-hyponormal. }
$$

In the cases where $W_{\alpha}$ is subnormal and $i:=\operatorname{rank}(\alpha)$, we have $\alpha=\left(\alpha_{0}, \cdots, \alpha_{2 i-2}\right)^{\wedge}$.

Proof. We only need to establish the sufficiency condition in (1.2.1). Let $i:=$ $\operatorname{rank}(\alpha)$. Since $W_{\alpha}$ is $i$-recursive, [CF1 Proposition 5.15] implies that the subnormality of $W_{\alpha}$ follows after we verify that $A(0, i-1) \geq 0$ and $A(1, i-1) \geq 0$. Now observe that $i-1 \leq\left[\frac{k}{2}\right]+1$ and

$$
A\left(j,\left[\frac{k}{2}\right]+1\right)=\left(\begin{array}{cc}
A(j, i-1) & * \\
* & *
\end{array}\right) \quad(j=0,1),
$$

so the positivity of $A(0, i-1)$ and $A(1, i-1)$ is a consequence of the $\left(\left[\frac{k}{2}\right]+1\right)$ hyponormality of $W_{\alpha}$. For the second assertion, observe that $\operatorname{det} A(n, i)=0$ for all $n \geq 0$. By assumption $A(n, i+1) \geq 0$, so by Lemma 1.1 (ii) we have $\operatorname{det} A(n, i+1)=$ 0 , which says that $\left(\alpha_{0}, \cdots, \alpha_{2 i-1}\right) \subset\left(\alpha_{0}, \cdots, \alpha_{2 i-2}\right)^{\wedge}$. By iteration we obtain $\left(\alpha_{0}, \cdots, \alpha_{k}\right) \subset\left(\alpha_{0}, \cdots, \alpha_{2 i-2}\right)^{\wedge}$, and therefore $\left(\alpha_{0}, \cdots, \alpha_{k}\right)^{\wedge}=\left(\alpha_{0}, \cdots, \alpha_{2 i-2}\right)^{\wedge}$. This proves the lemma. $k)$.

In what follows, and for notational convenience, we shall set $x_{-j}:=\alpha_{j}(0 \leq j \leq$ 
Theorem 1.3 (Subnormality Criterion). If $\alpha: x_{n}, \cdots, x_{1},\left(\alpha_{0}, \cdots, \alpha_{k}\right)^{\wedge}$, then

$$
W_{\alpha} \text { is subnormal } \Longleftrightarrow \begin{cases}W_{\alpha} \text { is }\left(\left[\frac{k+1}{2}\right]+1\right) \text {-hyponormal } & (n=1), \\ W_{\alpha} \text { is }\left(\left[\frac{k+1}{2}\right]+2\right) \text {-hyponormal } & (n>1) .\end{cases}
$$

Furthermore, in the cases where the above equivalence holds, if $\operatorname{rank}\left(\alpha_{0}, \cdots, \alpha_{k}\right)=$ $i$, then

$$
W_{\alpha} \text { is subnormal } \Longleftrightarrow \begin{cases}W_{\alpha} \text { is } i \text {-hyponormal } & (n=1), \\ W_{\alpha} \text { is }(i+1) \text {-hyponormal } & (n>1) .\end{cases}
$$

In fact,

$$
\left\{\begin{array}{c}
x_{1}=H_{i}\left(x_{0}, \cdots, x_{2-2 i}\right), \\
x_{2}=H_{i}\left(x_{1}, \cdots, x_{3-2 i}\right), \\
\left.\quad \ldots \cdots \cdots, x_{n-2 i}\right), \\
x_{n-1}=H_{i}\left(x_{n-2}, \cdots, x_{n-2 i+1}\right), \\
x_{n} \leq H_{i}\left(x_{n-1}, \cdots,\right.
\end{array}\right.
$$

where $H_{i}$ is the modulus of $i$-hyponormality [CF3, Proposition 3.4 and (3.4)]), i.e.,

$$
H_{i}(\alpha):=\sup \left\{x>0: W_{x \alpha} \text { is i-hyponormal }\right\} .
$$

Therefore, $W_{\alpha}=W_{x_{n}\left(x_{n-1}, \cdots, x_{n-2 i+1}\right)^{\wedge}}$.

Proof. Consider the $(k+1) \times(l+1)$ "Hankel" matrix $A(n ; k, l)$ defined by (cf. CL1]

$$
A(n ; k, l):=\left(\begin{array}{cccc}
\gamma_{n} & \gamma_{n+1} & \ldots & \gamma_{n+l} \\
\gamma_{n+1} & \gamma_{n+2} & \ldots & \gamma_{n+1+l} \\
\vdots & \vdots & & \vdots \\
\gamma_{n+k} & \gamma_{n+k+1} & \ldots & \gamma_{n+k+l}
\end{array}\right) \quad(n \geq 0)
$$

Case $1\left(\alpha: x_{1},\left(\alpha_{0}, \cdots, \alpha_{k}\right)^{\wedge}\right)$ : Let $\hat{A}(n ; k, l)$ and $A(n ; k, l)$ denote the Hankel matrices corresponding to the weight sequences $\left(\alpha_{0}, \cdots, \alpha_{k}\right)^{\wedge}$ and $\alpha$, respectively. Suppose $W_{\alpha}$ is $\left(\left[\frac{k+1}{2}\right]+1\right)$-hyponormal. Then by Lemma $1.2, W_{\left(\alpha_{0}, \cdots, \alpha_{k}\right)^{\wedge}}$ is subnormal. Observe that

$$
A(n+1 ; m, m)=x_{1}^{2} \hat{A}(n ; m, m) \text { for all } n \geq 0 \text { and all } m \geq 0 .
$$

Thus it suffices to show that $A(0 ; m, m) \geq 0$ for all $m \geq\left[\frac{k+1}{2}\right]+2$. Also observe that if $\tilde{B}$ denotes the $(k-1) \times k$ matrix obtained by eliminating the first row of a $k \times k$ matrix $B$, then

$$
\tilde{A}(0 ; m, m)=x_{1}^{2} \hat{A}(0 ; m-1, m) \quad \text { for all } m \geq\left[\frac{k+1}{2}\right]+2 .
$$

Therefore, for every $m \geq\left[\frac{k+1}{2}\right]+2, A(0 ; m, m)$ is a flat extension of

$$
A\left(0 ;\left[\frac{k+1}{2}\right]+1,\left[\frac{k+1}{2}\right]+1\right) .
$$

This implies $A(0 ; m, m) \geq 0$ for all $m \geq\left[\frac{k+1}{2}\right]+2$ and therefore $W_{\alpha}$ is subnormal.

Case $\left.2\left(\alpha: x_{n}, \cdots, x_{1},\left(\alpha_{0}, \cdots, \alpha_{k}\right)^{\wedge}\right)\right)$ : As in Case 1 , let $\hat{A}(n ; k, l)$ and $A(n ; k, l)$ denote the Hankel matrices corresponding to the weight sequences $\left(\alpha_{0}, \cdots, \alpha_{k}\right)^{\wedge}$ 
and $\alpha$, respectively. Observe that $\operatorname{det} \hat{A}\left(n ;\left[\frac{k+1}{2}\right]+1,\left[\frac{k+1}{2}\right]+1\right)=0$ for all $n \geq 0$. Suppose $W_{\alpha}$ is $\left(\left[\frac{k+1}{2}\right]+2\right)$-hyponormal. Observe that

$$
A\left(n+1 ;\left[\frac{k+1}{2}\right]+1,\left[\frac{k+1}{2}\right]+1\right)=x_{1}^{2} \cdots x_{n}^{2} \hat{A}\left(1 ;\left[\frac{k+1}{2}\right]+1,\left[\frac{k+1}{2}\right]+1\right),
$$

so that

$$
\operatorname{det} A\left(n+1 ;\left[\frac{k+1}{2}\right]+1,\left[\frac{k+1}{2}\right]+1\right)=0 .
$$

Also observe that

$$
A\left(n-1 ;\left[\frac{k+1}{2}\right]+2,\left[\frac{k+1}{2}\right]+2\right)=\left(\begin{array}{cc}
x_{2}^{2} \cdots x_{n}^{2} & * \\
* & A\left(n+1 ;\left[\frac{k+1}{2}\right]+1,\left[\frac{k+1}{2}\right]+1\right)
\end{array}\right) .
$$

Since $W_{\alpha}$ is $\left(\left[\frac{k+1}{2}\right]+1\right)$-hyponormal, it follows from Lemma 1.1 (iii) and (1.3.3) that $\operatorname{det} A\left(n-1 ;\left[\frac{k+1}{2}\right]+1,\left[\frac{k+1}{2}\right]+1\right)=0$. Note that

$$
A\left(n-1 ;\left[\frac{k+1}{2}\right]+1,\left[\frac{k+1}{2}\right]+1\right)=x_{1}^{2} \cdots x_{n}^{2}\left(\begin{array}{cccc}
\frac{1}{x_{1}^{2}} & \hat{\gamma}_{0} & \ldots & \hat{\gamma}_{\left[\frac{k+1}{2}\right]+1} \\
\hat{\gamma}_{0} & \hat{\gamma}_{1} & \ldots & \hat{\gamma}_{\left[\frac{k+1}{2}\right]+2} \\
\vdots & \vdots & & \vdots \\
\hat{\gamma}_{\left[\frac{k+1}{2}\right]+1} & \hat{\gamma}_{\left[\frac{k+1}{2}\right]+2} & \ldots & \hat{\gamma}_{2\left[\frac{k+1}{2}\right]+2}
\end{array}\right) \text {, }
$$

where $\hat{\gamma}_{j}$ denotes the moments corresponding to the weight sequence $\left(\alpha_{0}, \cdots, \alpha_{k}\right)^{\wedge}$. Therefore $x_{1}$ is determined uniquely by $\left\{\alpha_{0}, \cdots, \alpha_{k}\right\}$ such that $\left(x_{1}, \alpha_{0}, \cdots, \alpha_{k-1}\right)^{\wedge}$ $=x_{1},\left(\alpha_{0}, \cdots, \alpha_{k}\right)^{\wedge}$. More precisely, if $i:=\operatorname{rank}(\alpha)$ and $\varphi_{0}, \cdots, \varphi_{i-1}$ denote the coefficients of recursion in $\left(\alpha_{0}, \cdots, \alpha_{k}\right)^{\wedge}$, then

$$
x_{1}=H_{i}\left[\left(\alpha_{0}, \cdots, \alpha_{k}\right)^{\wedge}\right]=\left[\frac{\varphi_{0}}{\hat{\gamma}_{i-1}-\varphi_{i-1} \hat{\gamma}_{i-2}-\cdots-\varphi_{1} \hat{\gamma}_{0}}\right]^{\frac{1}{2}}
$$

(cf. CF3, (3.4)]). Continuing this process we can see that $x_{1}, \cdots, x_{n-1}$ are determined uniquely by a telescoping method such that

$$
\left(x_{n-1}, \cdots, x_{n-1-k}\right)^{\wedge}=x_{n-1}, \cdots, x_{1},\left(\alpha_{0}, \cdots, \alpha_{k}\right)^{\wedge}
$$

and $W_{\left(x_{n-1}, \cdots, x_{n-1-k}\right)^{\wedge}}$ is subnormal. Therefore, after $(n-1)$ steps, Case 2 reduces to Case 1. This completes the proof of the first assertion. For the second assertion, note that if $\operatorname{rank}\left(\alpha_{0}, \cdots, \alpha_{k}\right)=i$, then

$$
\operatorname{det} \hat{A}(n ; i, i)=0 \text {. }
$$

Now applying the above argument with $i$ in place of $\left[\frac{k+1}{2}\right]+1$ gives that $x_{1}, \cdots, x_{n-1}$ are determined uniquely by $\alpha_{0}, \cdots, \alpha_{2 i-2}$ such that $W_{\left(x_{n-1}, \cdots, x_{n-2 i-1}\right)^{\wedge}}$ is subnormal. Thus the second assertion immediately follows. Finally, observe that the preceding argument also establishes the remaining assertions.

Remark 1.4. (a) From Theorem 1.3 we note that the subnormality of an extension of a recursive shift is independent of its length, if the length is bigger than 1.

(b) In Theorem 1.3, "[ $\left.\frac{k+1}{2}\right]$ " cannot be relaxed to "[ $\left[\frac{k}{2}\right]$ ". For example, consider the following weight sequences:

(i) $\alpha: \sqrt{\frac{1}{2}},\left(\sqrt{\frac{3}{2}}, \sqrt{3}, \sqrt{\frac{10}{3}}, \sqrt{\frac{17}{5}}\right)^{\wedge}$ with $\varphi_{0}=0$;

(ii) $\alpha^{\prime}: \sqrt{\frac{1}{2}}, \sqrt{\frac{3}{2}},\left(\sqrt{3}, \sqrt{\frac{10}{3}}, \sqrt{\frac{17}{5}}\right)^{\wedge}$. 
Observe that $\alpha$ equals $\alpha^{\prime}$. Then a straightforward calculation shows that $W_{\alpha}$ (and hence $W_{\alpha^{\prime}}$ ) is 2-hyponormal but not 3-hyponormal (and hence, not subnormal). Note that $k=3$ and $n=1$ in (i) and $k=2$ and $n=2$ in (ii).

(c) Note that the second assertion of Theorem 1.3 does not imply that if $\operatorname{rank}\left(\alpha_{0}, \cdots, \alpha_{k}\right)=i$, then (1.3.2) holds in general. Theorem 1.3 says only that when $W_{\alpha}$ is $\left(\left[\frac{k+1}{2}\right]+1\right)$-hyponormal $(n=1), i$-hyponormality and subnormality coincide, and that when $W_{\alpha}$ is $\left(\left[\frac{k+1}{2}\right]+2\right)$-hyponormal $(n>1),(i+1)$-hyponormality and subnormality coincide. For example consider the weight sequence

$$
\left.\hat{\alpha} \equiv\left(\sqrt{2}, \sqrt{3}, \sqrt{\frac{10}{3}}, \sqrt{\frac{17}{5}}, 2\right)^{\wedge} \text { with } \varphi_{0}=0 \text { (here } \varphi_{1}=0 \text { also }\right) .
$$

Since $\left(\sqrt{2}, \sqrt{3}, \sqrt{\frac{10}{3}}, \sqrt{\frac{17}{5}}\right) \subset\left(\sqrt{2}, \sqrt{3}, \sqrt{\frac{10}{3}}\right)^{\wedge}$, we can see that $\operatorname{rank}(\alpha)=2$. Put

$$
\beta \equiv 1,\left(\sqrt{2}, \sqrt{3}, \sqrt{\frac{10}{3}}, \sqrt{\frac{17}{5}}, 2\right)^{\wedge} .
$$

If (1.3.2) held true without assuming (1.3.1), then 2-hyponormality would imply subnormality for $W_{\beta}$. However, a straightforward calculation shows that $W_{\beta}$ is 2hyponormal but not 3-hyponormal (and hence not subnormal). In fact, $\operatorname{det} A(n, 2)$ $=0$ for all $n \geq 0$ except for $n=2$ and $\operatorname{det} A(2,2)=160>0$, while since

$$
\varphi_{3}=-\frac{\alpha_{3}^{2} \alpha_{4}^{2}\left(\alpha_{5}^{2}-\alpha_{4}^{2}\right)}{\alpha_{4}^{2}-\alpha_{3}^{2}}=-102 \quad \text { and } \quad \varphi_{4}=\frac{\alpha_{4}^{2}\left(\alpha_{5}^{2}-\alpha_{3}^{2}\right)}{\alpha_{4}^{2}-\alpha_{3}^{2}}=34
$$

(so that $\alpha_{6}=\sqrt{\varphi_{4}-\frac{\varphi_{3}}{\alpha_{5}^{2}}}=\sqrt{\frac{17}{2}}$ ), we have that

$$
\operatorname{det} A(1,3)=\operatorname{det}\left(\begin{array}{cccc}
1 & 2 & 6 & 20 \\
2 & 6 & 20 & 68 \\
6 & 20 & 68 & 272 \\
20 & 68 & 272 & 2312
\end{array}\right)=-3200<0 .
$$

(d) On the other hand, Theorem 1.3 does show that if $\alpha \equiv\left(\alpha_{0}, \cdots, \alpha_{k}\right)$ is such that $\operatorname{rank}(\alpha)=i$ and $W_{\hat{\alpha}}$ is subnormal with associated Berger measure $\mu$, then $W_{\hat{\alpha}}$ has an $n$-step $(i+1)$-hyponormal extension $W_{x_{n}, \cdots, x_{1}, \hat{\alpha}}(n \geq 2)$ if and only if $\frac{1}{t^{n}} \in L^{1}(\mu)$

$$
x_{j+1}=\left[\frac{\varphi_{0}}{\gamma_{i-1}^{(j)}-\varphi_{i-1} \gamma_{i-2}^{(j)}-\cdots-\varphi_{1} \gamma_{0}^{(j)}}\right]^{\frac{1}{2}} \quad(0 \leq j \leq n-2),
$$

and

$$
x_{n} \leq\left[\frac{\varphi_{0}}{\gamma_{i-1}^{(n-1)}-\varphi_{i-1} \gamma_{i-2}^{(n-1)}-\cdots-\varphi_{1} \gamma_{0}^{(n-1)}}\right]^{\frac{1}{2}},
$$

where $\varphi_{0}, \cdots, \varphi_{i-1}$ denote the coefficients of recursion in $\left(\alpha_{0}, \cdots, \alpha_{2 i-2}\right)^{\wedge}$ and $\gamma_{m}^{(j)}(0 \leq m \leq i-1)$ are the moments corresponding to the weight sequence $\left(x_{j}, \cdots, x_{1}, \alpha_{0}, \cdots, \alpha_{k-j}\right)^{\wedge}$ with $\gamma_{m}^{(0)}=\gamma_{m}$.

We now observe that the determination of $k$-hyponormality and subnormality for canonical rank-one perturbations of recursive shifts falls within the scope of the theory of extensions. 
Corollary 1.5. Let $\alpha \equiv\left\{\alpha_{n}\right\}_{n=0}^{\infty}=\left(\alpha_{0}, \cdots, \alpha_{k}\right)^{\wedge}$. If $W_{\alpha^{\prime}}$ is a perturbation of $W_{\alpha}$ at the $j$-th weight, then

$$
W_{\alpha^{\prime}} \text { is subnormal } \Longleftrightarrow \begin{cases}W_{\alpha^{\prime}} \text { is }\left(\left[\frac{k+1}{2}\right]+1\right) \text {-hyponormal } & (j=0), \\ W_{\alpha^{\prime}} \text { is }\left(\left[\frac{k+1}{2}\right]+2\right) \text {-hyponormal } & (j \geq 1) .\end{cases}
$$

Proof. Observe that if $j=0$, then $\alpha^{\prime}=x,\left(\alpha_{1}, \cdots, \alpha_{k+1}\right)^{\wedge}$ and if instead $j \geq 1$, then $\alpha^{\prime}=\alpha_{0}, \cdots, \alpha_{j-1}, x,\left(\alpha_{j+1}, \cdots, \alpha_{j+k+1}\right)^{\wedge}$. Thus the result immediately follows from Theorem 1.3.

\section{Extremality of ReCURSively Generated Shifts}

In Corollary 1.5, we showed that if $\alpha(x)$ is a canonical rank-one perturbation of a recursive weight sequence, then subnormality and $k$-hyponormality for the corresponding shift eventually coincide. In this section we consider a converse.

Problem 2.1 (Extremality Problem). Let $\alpha(x)$ be a canonical rank-one perturbation of a weight sequence $\alpha$. If there exists $k \geq 1$ such that $(k+1)$-hyponormality and $k$-hyponormality for the corresponding shift $W_{\alpha(x)}$ coincide, does it follow that $\alpha(x)$ is recursively generated?

In [CF3, the following extremality criterion was established.

Lemma 2.2 (Extremality Criterion [CF3, Theorem 5.12, Proposition 5.13]). Let $\alpha$ be a weight sequence and let $k \geq 1$.

(i) If $W_{\alpha}$ is $k$-extremal (i.e., $\operatorname{det} A(j, k)=0$ for all $\left.j \geq 0\right)$, then $W_{\alpha}$ is recursive subnormal.

(ii) If $W_{\alpha}$ is k-hyponormal and if $\operatorname{det} A\left(i_{0}, j_{0}\right)=0$ for some $i_{0} \geq 0$ and some $j_{0}<k$, then $W_{\alpha}$ is recursive subnormal.

In particular, Lemma 2.2 (ii) shows that if $W_{\alpha}$ is subnormal and if $\operatorname{det} A\left(i_{0}, j_{0}\right)=$ 0 for some $i \geq 0$ and some $j \geq 0$, then $W_{\alpha}$ is recursive subnormal.

We now answer Problem 2.1 affirmatively.

Theorem 2.3. Let $\alpha \equiv\left\{\alpha_{n}\right\}_{n=0}^{\infty}$ be a weight sequence and let $\alpha_{j}(x)$ be a canonical perturbation of $\alpha$ in the $j$-th weight. Write

$$
\mathfrak{H}_{k}:=\left\{x \in \mathbb{R}^{+}: W_{\alpha_{j}(x)} \text { is k-hyponormal }\right\} .
$$

If $\mathfrak{H}_{k}=\mathfrak{H}_{k+1}$ for some $k \geq 1$, and if $x \in \mathfrak{H}_{k}$, then $\alpha_{j}(x)$ is recursively generated, i.e., $W_{\alpha_{j}(x)}$ is recursive subnormal.

Proof. Suppose $\mathfrak{H}_{k}=\mathfrak{H}_{k+1}$ and let $H_{k}:=\sup _{x} \mathfrak{H}_{k}$. To avoid triviality we assume $\alpha_{j-1}<x<\alpha_{j+1}$.

Case $1(j=0)$ : In this case, clearly $H_{k}^{2}$ is the nonzero root of the equation $\operatorname{det} A(0, k)=0$ and for $x \in\left(0, H_{k}\right], W_{\alpha_{0}(x)}$ is $k$-hyponormal. By assumption $H_{k}=$ $H_{k+1}$, so $W_{\alpha_{0}\left(H_{k+1}\right)}$ is $(k+1)$-hyponormal. The result now follows from Lemma 2.2 (ii).

Case $2(j \geq 1)$ : Let $A_{x}(n, k)$ denote the Hankel matrix corresponding to $\alpha_{j}(x)$. Since $W_{\alpha_{j}(x)}$ is $(k+1)$-hyponormal for $x \in \mathfrak{H}_{k}$, we have that $A_{x}(n, k+1) \geq 0$ for all $n \geq 0$ and all $x \in \mathfrak{H}_{k}$. Observe that if $n \geq j+1$, then

$$
A_{x}(n, k)=\alpha_{0}^{2} \cdots \alpha_{j-1}^{2} x^{2}\left(\begin{array}{ccc}
\tilde{\gamma}_{n-j-1} & \ldots & \tilde{\gamma}_{n-j-1+k} \\
\vdots & & \vdots \\
\tilde{\gamma}_{n-j-1+k} & \ldots & \tilde{\gamma}_{n-j-1+2 k}
\end{array}\right),
$$


where $\tilde{\gamma}_{*}$ denotes the moments corresponding to the subsequence $\alpha_{j+1}, \alpha_{j+2}, \cdots$. Therefore for $n \geq j+1$, the positivity of $A_{x}(n, k)$ is independent of the values of $x>0$. This gives

$$
W_{\alpha_{j}(x)} \text { is } k \text {-hyponormal } \Longleftrightarrow A_{x}(n, k) \geq 0 \text { for all } n \leq j .
$$

Write

$$
\mathfrak{H}_{k}^{(i)}:=\left\{x: \operatorname{det} A_{x}(i, k) \geq 0 \text { and } \alpha_{j-1}<x<\alpha_{j+1}\right\} \quad(0 \leq i \leq j)
$$

and

$$
H_{k}^{(i)}=\sup _{x} \mathfrak{H}_{k}^{(i)} \quad(0 \leq i \leq j) .
$$

Since $\operatorname{det} A_{x}(i, k)$ is a polynomial in $x$ we have $\operatorname{det} A_{H_{k}^{(i)}}(i, k)=0$. Observe that

$$
\bigcap_{i=0}^{j} \mathfrak{H}_{k}^{(i)}=\mathfrak{H}_{k} \quad \text { and } \quad \max _{0 \leq i \leq j} H_{k}^{(i)}=H_{k} .
$$

Since $\mathfrak{H}_{k}$ is a closed interval, by [CL2, Theorem 2.11] it follows that $H_{k} \in \mathfrak{H}_{k}$, say $H_{k}=H_{k}^{(p)}$ for some $0 \leq p \leq j$. Then $\operatorname{det} A_{H_{k}^{(p)}}(p, k)=0$ and $W_{\alpha\left(H_{k}^{(p)}\right)}$ is $(k+1)$-hyponormal. Therefore it follows from Lemma 2.2 (ii) that $W_{\alpha}$ is recursive subnormal. This completes the proof.

We conclude this section with two corollaries of independent interest.

Corollary 2.4. With the notations in Theorem 2.3, if $j \geq 1$ and $\mathfrak{H}_{k}=\mathfrak{H}_{k+1}$ for some $k$, then $\mathfrak{H}_{k}$ is a singleton set.

Proof. By CL2, Theorem 2.2],

$$
\mathfrak{H}_{\infty}:=\left\{x \in \mathbb{R}^{+}: W_{\alpha_{j}(x)} \text { is subnormal }\right\}
$$

is a singleton set. By Theorem 2.3, we have that $\mathfrak{H}_{k}=\mathfrak{H}_{\infty}$.

Corollary 2.5. If $W_{\alpha}$ is a nonrecursive shift with weight sequence $\alpha=\left\{\alpha_{n}\right\}_{n=0}^{\infty}$ and if $\alpha(x)$ is a canonical rank-one perturbation of $\alpha$, then for every $k \geq 1$ there always exists a gap between $k$-hyponormality and $(k+1)$-hyponormality for $W_{\alpha(x)}$. More concretely, if we let

$$
\mathfrak{H}_{k}:=\left\{x: W_{\alpha(x)} \text { is k-hyponormal }\right\},
$$

then $\left\{\mathfrak{H}_{k}\right\}_{k=1}^{\infty}$ is a strictly decreasing nested sequence of closed intervals in $(0, \infty)$ except when the perturbation occurs in the first weight. In that case, the intervals are of the form $\left(0, H_{k}\right]$.

Proof. Straightforward from Theorem 2.3.

\section{Some ReVealing EXAmples}

We now illustrate our results with two examples. Consider $\alpha(y, x): \sqrt{y}, \sqrt{x}$, $(\sqrt{a}, \sqrt{b}, \sqrt{c})^{\wedge}$, where $a<b<c$. Without loss of generality, we assume $a=1$. Observe that

$$
H_{2}(1, \sqrt{b}, \sqrt{c})=\sqrt{\frac{b c-b^{2}}{1+b c-2 b}}
$$


and

$$
\left(H_{2}(\sqrt{x}, 1, \sqrt{b})\right)^{2}=\frac{x(b-1)}{(x-1)^{2}+(b-1)}:=f(x) .
$$

Thus $W_{\alpha(y, x)}$ is 2-hyponormal if and only if $0<x \leq \frac{b c-b^{2}}{1+b c-2 b}$ and $0<y \leq f(x)$. To completely describe the region $\mathcal{R}:=\left\{(x, y): W_{\alpha(y, x)}\right.$ is 2 -hyponormal $\}$, we study the graph of $f$. Observe that

$$
f^{\prime}(x)=\frac{(b-1)\left(b-x^{2}\right)}{\left(b-2 x+x^{2}\right)^{2}}>0 \quad \text { and } \quad f^{\prime \prime}(x)=\frac{2(b-1)\left(2 b-3 b x+x^{3}\right)}{\left(b-2 x+x^{2}\right)^{3}} .
$$

Note that $b-2 x+x^{2}=(b-1)+(1-x)^{2}>0$ and $f^{\prime}(\sqrt{b})=0$. To consider the sign of $f^{\prime \prime}$, we let $g(x):=2 b-3 b x+x^{3}$. Then $g^{\prime}(\sqrt{b})=0, g(0)=2 b>0$, $g(1)=-b+1<0$, and $g^{\prime \prime}(x)>0(x>0)$. Hence there exists $x_{0} \in(0,1)$ such that $f^{\prime \prime}\left(x_{0}\right)=0, f^{\prime \prime}(x)>0$ on $0<x<x_{0}$, and $f^{\prime \prime}(x)<0$ on $x_{0}<x \leq 1$. We investigate which of the two values $x_{0}$ or $\widetilde{H}:=H_{2}(1, \sqrt{b}, \sqrt{c})^{2}$ is bigger. By a simple calculation, we have

$$
g(\widetilde{H})=\frac{(-1+b) b \cdot g_{1}(b, c)}{(1-2 b+b c)^{3}}
$$

where

$$
g_{1}(b, c)=-\left(2-10 b+17 b^{2}-11 b^{3}+b^{4}+3 b c-9 b^{2} c+9 b^{3} c-3 b^{3} c^{2}+b^{2} c^{3}\right) .
$$

For notational convenience we let $b:=1+h, c:=1+h+k$. Then

$$
g_{1}(b, c)=2 h^{5}+\left(3 h^{3}+3 h^{4}\right) k+\left(-1-2 h-h^{2}\right) k^{3} .
$$

If $h$ is sufficiently small (i.e., $b$ is sufficiently close to 1 ), then $g_{1}<0$, i.e., $\widetilde{H}>x_{0}$. If $k$ is sufficiently small (i.e., $b$ is sufficiently close to $c$ ), then $g_{1}>0$, i.e., $\widetilde{H}<x_{0}$. Thus, if $\widetilde{H} \leq x_{0}$, then $f$ is concave up on $x \leq \widetilde{H}$. If $\widetilde{H}>x_{0}$, then $\left(x_{0}, f\left(x_{0}\right)\right)$ is an inflection point. Thus, $f$ is concave up on $0<x<x_{0}$ and concave down on $x_{0}<x \leq \widetilde{H}$. Moreover, $W_{\alpha(y, x)}$ is 2-hyponormal if and only if $(x, y) \in\{(x, y) \mid 0 \leq$ $y \leq f(x), 0<x \leq \widetilde{H}\}$, and $W_{\alpha(y, x)}$ is $k$-hyponormal $(k \geq 3)$ if and only if $x=\widetilde{H}$ and $0 \leq y \leq f(\widetilde{H})$.

Example 3.1 $(b=2, c=3)$.

$$
f(x)=\frac{x}{1+(1-x)^{2}} .
$$

The graph of $\mathcal{R}$ is given in Figure 1; notice that $f$ is concave up in this case.

Example $3.2\left(b=\frac{11}{10}, c=10\right)$.

$$
f(x)=\frac{x}{11-20 x+10 x^{2}} .
$$

The graph of $\mathcal{R}$ is given in Figure 2; in this case, $f$ has an inflection point at $x_{0} \approx 0.85821$.

\section{ACKNowledgement}

This paper was written while the second- and/or the third-named authors visited the Department of Mathematics at The University of Iowa during the winter breaks of 1998 and 1999, and the summer break of 1999. They wish to thank the faculty members in that unit for their warm hospitality. 


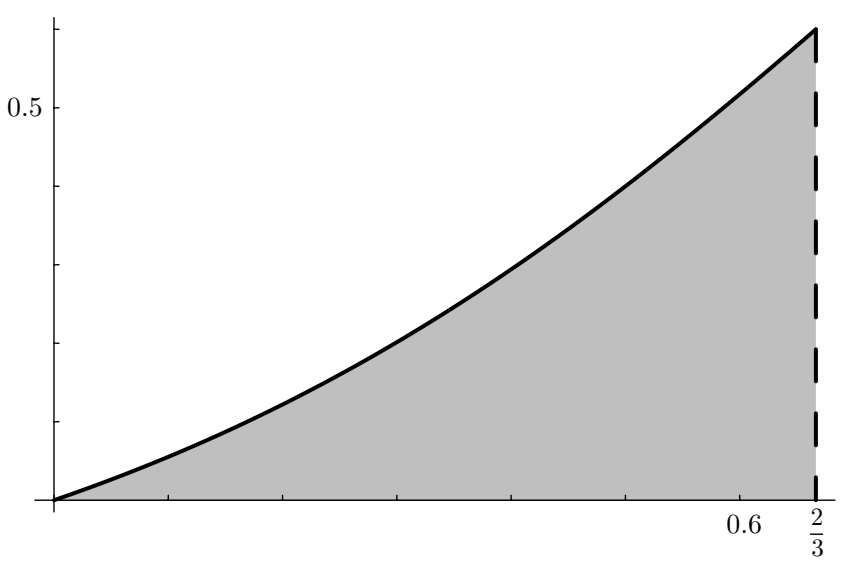

FIGURE 1.

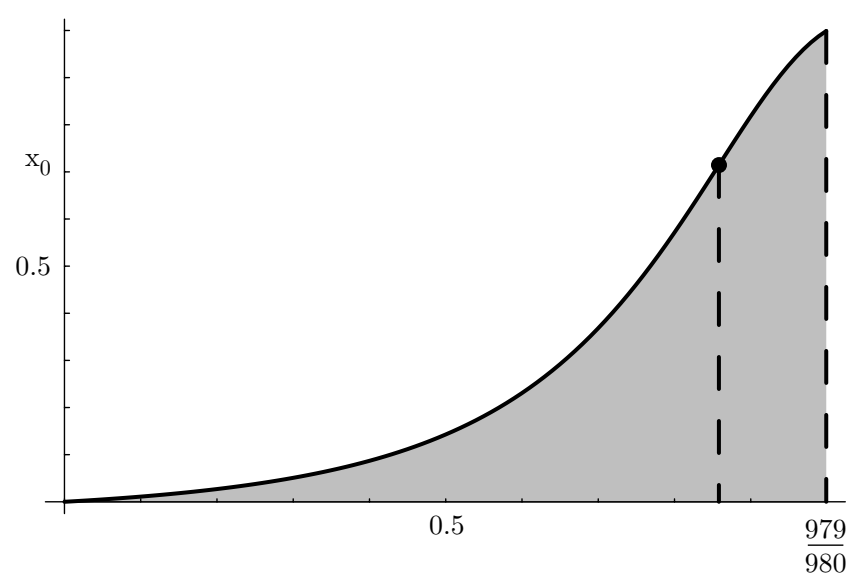

FIGURE 2.

\section{REFERENCES}

[At] A. Athavale, On joint hyponormality of operators, Proc. Amer. Math. Soc. 103 (1988), 417-423. MR 89f:47033

[Br] J. Bram, Subnormal operators, Duke Math. J. 22 (1955), 75-94. MR 16:835a

[Con] J.B. Conway, Subnormal Operators, Research Notes in Mathematics, vol. 51, Pitman Publ. Co., London, 1981. MR 83i:47030

[Cu1] R.E. Curto, Quadratically hyponormal weighted shifts, Int. Eq. Op. Th. 13 (1990), 49-66. MR 90k:47061

[Cu2] J Joint hyponormality:A bridge between hyponormality and subnormality, Operator Theory: Operator Algebras and Applications (Durham, NH, 1988) (W.B. Arveson and R.G. Douglas, eds.), Proc. Sympos. Pure Math., vol. 51, part II, American Mathematical Society, Providence, (1990), Part 11, 69-91. MR 91k:47049

[CF1] R.E. Curto and L.A. Fialkow, Recursiveness, positivity, and truncated moment problems, Houston J. Math. 17 (1991), 603-635. MR 93a:47016

[CF2] , Recursively generated weighted shifts and the subnormal completion problem, Int. Eq. Op. Th. 17 (1993), 202-246. MR 94h:47050

[CF3] - Recursively generated weighted shifts and the subnormal completion problem, II, Int. Eq. Op. Th. 18 (1994), 369-426. MR 94m:47044 
[CL1] R.E. Curto and W.Y. Lee, Joint hyponormality of Toeplitz pairs, Memoirs Amer. Math. Soc. 150 , no. 712 (2001), x+65 pages.

[CL2] , Flatness, perturbations and completions of weighted shifts, preprint 1999.

[CMX] R.E. Curto, P.S. Muhly and J. Xia, Hyponormal pairs of commuting operators, Contributions to Operator Theory and Its Applications (Mesa, AZ, 1987) (I. Gohberg, J.W. Helton and L. Rodman, eds.), Operator Theory: Advances and Applications, vol. 35, Birkhäuser, Basel-Boston, (1988), 1-22. MR 90m:47037

[Hal] P.R. Halmos, A Hilbert Space Problem Book, 2nd ed., Springer, New York, 1982. MR 84e:47001

[McCP] S. McCullough and V. Paulsen, A note on joint hyponormality, Proc. Amer. Math. Soc. 107 (1989), 187-195. MR 90a:47062

[Shi] A. Shields, Weighted shift operators and analytic function theory, Math. Surveys 13 (1974), 49-128. MR 50:14341

[Sta] J. Stampfli, Which weighted shifts are subnormal, Pacific J. Math. 17 (1966), 367-379. MR 33:1740

Department of Mathematics, University of Iowa, Iowa City, Iowa 52242

E-mail address: curto@math.uiowa.edu

Department of Mathematics, Kyungpook National University, Taegu 702-701, Korea

E-mail address: ibjung@bh.kyungpook.ac.kr

Department of Mathematics, Sungkyunkwan University, Suwon 440-746, Korea

E-mail address: wylee@yurim.skku.ac.kr 